\title{
Readmission predicts 90-day mortality after esophagectomy: Analysis of Surveillance, Epidemiology, and End Results Registry linked to Medicare outcomes
}

\author{
Yinin $\mathrm{Hu}, \mathrm{MD},{ }^{\mathrm{a}}$ Timothy L. McMurry, PhD, ${ }^{\mathrm{b}}$ George J. Stukenborg, $\mathrm{PhD},{ }^{\mathrm{b}}$ and \\ Benjamin D. Kozower, MD, MPH ${ }^{\mathrm{a}, \mathrm{b}}$
}

\begin{abstract}
Objectives: Postoperative readmission is an increasingly scrutinized quality metric that affects patient satisfaction and cost. Even more important is its implication for short-term prognosis. The purpose of this study is to characterize postesophagectomy readmissions and determine their relationship with subsequent 90-day mortality.

Methods: Data were extracted for esophagectomy patients from the linked SEER-Medicare Registry (2000-2009), which provides longitudinal information about Medicare beneficiaries who have cancer. We assessed demographics, comorbidities, 30-day readmission, and 90-day mortality. Readmitting facility and diagnoses were identified. A hierarchic multivariable regression model clustered at the hospital level assessed the relationship between readmission within 30 days of discharge and 90-day mortality.

Results: We identified 1543 patients discharged alive after esophagectomy. Among patients discharged alive, the readmission rate was 319 of 1543 $(20.7 \%) ; 107$ of $319(33.5 \%)$ readmissions were to facilities that did not perform the index operation. Mortality rate at 90 days among patients discharged alive was 98 of $1543(6.4 \%)$. Readmission was associated with a 4-fold increase in mortality $(16.3 \%$ vs $3.8 \%, P<.001)$. Using multivariable regression, readmission was the strongest predictor of mortality (odds ratio 6.64, $P<.001$ ), with a stronger association than age, Charlson score, and index length of stay. Readmission diagnoses with the highest mortality rates were those associated with pulmonary, gastrointestinal, and cardiovascular diagnoses.
\end{abstract}

Conclusions: Patients readmitted within 30 days of discharge after esophagectomy are at exceptionally high risk for early mortality. Early recognition of life-threatening readmission diagnoses is essential to providing optimal care. (J Thorac Cardiovasc Surg 2015;150:1254-60)

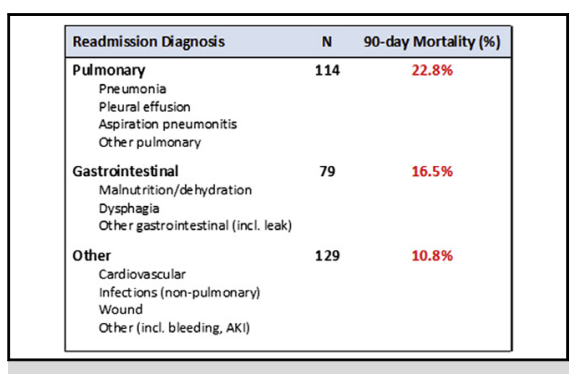

Readmission diagnosis groups and associated risks of 90-day mortality.

\section{Central Message}

One in 5 esophagectomy patients are readmitted early after discharge. Readmitted patients have a 5 -fold increase in early mortality.

\section{Perspective}

Early readmissions are common after esophagectomy. This study assesses temporal trends in readmissions, the most common readmitting diagnoses, and the relationships between readmission and 90-day mortality. Results highlight the clinical acuity and poor prognosis of readmitted patients, and they argue for early rootcause exploration and aggressive care.

See Editorial page 1030.
Esophageal cancer is the fastest-growing cancer in the United States. ${ }^{1}$ For appropriate surgical candidates, esophagectomy is the principal treatment for early cancer

From the a Division of Thoracic Surgery/Department of Surgery, and ${ }^{\mathrm{b}}$ Department of Public Health Sciences, University of Virginia Health System, Charlottesville, Va. Funding support was provided by the Agency for Healthcare Research and Quality (K080HS18049, to B.D.K.) and by the National Institutes of Health (T32 CA163177, to Y.H.).

Received for publication May 13, 2015; revisions received July 23, 2015; accepted for publication Aug 19, 2015; available ahead of print Sept 25, 2015.

Address for reprints: Benjamin D. Kozower, MD, MPH, University of Virginia Health System, General Thoracic Surgery, P.O. Box 800679, Charlottesville, VA 22908-0679 (E-mail: bdk8g@virginia.edu).

$0022-5223 / \$ 36.00$

Copyright (C) 2015 by The American Association for Thoracic Surgery

http://dx.doi.org/10.1016/j.jtcvs.2015.08.071 and a vital component of multimodal therapy for locally advanced disease. However, despite advancements in operative approach and postoperative care, esophagectomy for cancer remains a morbid operation, ${ }^{2-4}$ with early postoperative mortality ranging from $1 \%$ to $13 \%$. Methods to reduce this risk have been under intensive investigation during the past 15 years, with most studies focusing on mortality risk stratification, assessment of less-invasive and minimally invasive approaches, the volume-outcome relationship, and formalized postoperative management pathways. $^{5-7}$

Although the association between major surgical complications and mortality is widely recognized, using surgical complications as a quality metric is problematic 


\author{
Abbreviations and Acronyms \\ $\mathrm{CI}=$ confidence interval \\ ICD-9 $=$ International Classification of Diseases \\ $\mathrm{IQR}=$ interquartile range \\ LOS = length of stay \\ $\mathrm{OR}=$ odds ratio \\ SEER $=$ Surveillance, Epidemiology, and End \\ Results
}

because of the subjectivity of the definitions used. ${ }^{8}$ Data from the American College of Surgeons National Surgical Quality Improvement Program indicate that the primary difference between high- and low-mortality providers is not the incidence of surgical complications, but rather the mortality rates associated with these complications. ${ }^{9}$ Evidently, focus should be placed on not only avoiding complications, but also on the proper management of complications that do occur.

Due to the ease of accessing early readmission data through national databases, this factor is increasingly being recognized for its clinical and financial significance..$^{10,11}$ The readmission rate after esophagectomy is reportedly ${ }^{12-14}$ between $5 \%$ and $20 \%$. Although readmission has been linked to early postoperative mortality in colorectal, hepatobiliary, and pulmonary surgery, its impact on early mortality after esophagectomy is underexplored. ${ }^{11,15,16}$

Although initial readmitting diagnoses may not reflect the true root cause of a patient's demise, these early indicators may represent opportunities to appropriately address potentially life-threatening conditions. The purpose of this study is to better describe the characteristics and downstream effects of readmissions that occur within 30 days of discharge after esophagectomy. In particular, we sought to determine the temporal trends in postoperative readmissions, the most common readmitting diagnoses, and the relationships between readmission and subsequent 90-day mortality. We hypothesized that readmission is associated with an increased risk of death, with certain readmission diagnoses having higher mortality rates than others.

\section{METHODS}

The Surveillance, Epidemiology, and End Results (SEER) Registry is a population-based collection of incident cases with cancer diagnostic, descriptive, and therapeutic information. The Medicare healthcare claims database includes survival data, as well as information abstracted from discharge records of inpatient admissions, outpatient care claims, and other healthcare event claims for which Medicare provides reimbursement. The National Cancer Institute links the SEER Registry with the Medicare claims database, which includes records for $93 \%$ of patients aged $\geq 65$ years, who make up $24 \%$ of the total population. ${ }^{17}$ This combined database accurately records postoperative readmissions, including those at facilities that did not provide the index operation. Unlike the database of the Society of Thoracic Surgeons, the SEER Registry linked with Medicare (SEER-Medicare) includes long-term follow-up of patient survival, thereby providing an opportunity for longitudinal studies of mortality among cancer patients that are broadly generalizable to the Medicare population.

The 2000 to 2009 SEER-Medicare database was used to identify records for all patients aged $\geq 66$ years who had esophageal cancer of any histologic type and stage who received surgical resection. Patients enrolled in a Medicare health maintenance organization were excluded. Additional exclusionary criteria included esophageal cancer diagnosed during autopsy, missing date of diagnosis, and in-hospital mortality during the operative admission. To ensure adequate records of pre-existing comorbidities, we additionally excluded patients who were ineligible for Medicare during the 3 months preceding surgery.

Demographic information included age, gender, and the operative and readmitting facilities. Clinical data included year of operation, final pathologic stage, extent of resection, and preoperative comorbidities. Because the SEER-Medicare database does not separate preoperative from postoperative diagnoses for a given admission, early postoperative complications could not be distinguished from preexisting morbidities.

To determine causes for readmission, the first 2 International Classification of Diseases, 9th edition (ICD-9), diagnosis codes associated with each readmission were collected. If diagnosis codes belonging to $>1$ organ system are associated with 1 readmission, investigator judgement was used to determine the primary readmitting etiology. Comorbidities present prior to the index operation were identified based on the Deyo modification of the Charlson index, ${ }^{18}$ using criteria provided by the National Cancer Institute for use with inpatient files, physician claims data, and outpatient files. ${ }^{19,20}$ Preoperative induction treatment with either chemotherapy alone or chemoradiation was noted. Mortality measures were derived from Medicare death certificate records. The primary outcomes were readmission within 30 days of discharge and mortality within 90 days of surgery.

Ninety-day mortality was compared for readmitted versus non-readmitted patients using $\chi^{2}$ analysis. Subgroup comparisons were used to assess mortality rates based on the readmitting facility (operative vs nonoperative), and readmitting diagnoses were grouped by organ system. Reporting of SEER-Medicare data requires a minimum cell size of $>11$ patients, for confidentiality considerations. Thus, diagnosisspecific mortality rates are not presented, owing to limitations on reportable cell size.

A hierarchic generalized linear model was created to estimate the risk of readmission using clinically relevant variables, including age, Charlson score, gender, operative year, induction therapy, discharge disposition, and postoperative length of stay (LOS). ${ }^{21}$ Because the relationship between LOS and readmission is not linear, this variable was modeled using a linear spline with a knot at 20 days. To determine the impact of readmission on mortality, a second model was created for 90-day mortality with the addition of 30-day readmission as a predictive variable.

Both models were adjusted for data clustered by hospital provider. Model predictors were selected a priori, based on frequency of occurrence within our dataset and review of recent thoracic surgery literature. The statistical significance of each predictor was assessed using the $\mathrm{F}$ test. Test for provider covariance determined whether risk-adjusted readmission rates and mortality rates varied significantly across hospitals.

To determine the relationship between index postoperative LOS and likelihood of readmission, a third regression model was generated using natural cubic splines. This method allowed the interpretation of a nonlinear relationship, and controlled for patient age, Charlson comorbidity index, and induction therapy. Spline knots were placed at 9, 11, 14, 18, and 25 days, with boundary knots at 7 and 29 days; results were similar at the various knot placements. All analyses were performed using SAS (version 9.4; SAS Institute, Inc, Cary, NC) and R statistical 
TABLE 1. Patient demographics

\begin{tabular}{|c|c|c|c|}
\hline Demographics & $\begin{array}{c}\text { Readmission, } \\
\text { n ( } \%)\end{array}$ & $\begin{array}{c}\text { No readmission, } \\
\mathbf{n}(\%)\end{array}$ & $\begin{array}{c}P \\
\text { value }\end{array}$ \\
\hline \multicolumn{4}{|l|}{ Age $(y)$} \\
\hline $65-69$ & $110(34)$ & $422(34)$ & .916 \\
\hline $70-74$ & $102(32)$ & $410(34)$ & \\
\hline $75-79$ & $71(22)$ & $249(20)$ & \\
\hline $80-84$ & $24(8)$ & $119(10)$ & \\
\hline$\geq 85$ & $12(4)$ & $24(2)$ & \\
\hline Gender. female & $71(22)$ & $228(19)$ & .726 \\
\hline \multicolumn{4}{|l|}{ Year of diagnosis } \\
\hline $2000-2001$ & $57(18)$ & $219(18)$ & .983 \\
\hline $2002-2003$ & $59(18)$ & $241(20)$ & \\
\hline 2004-2005 & $74(23)$ & $295(24)$ & \\
\hline 2006-2007 & $65(20)$ & $258(21)$ & \\
\hline 2008-2009 & $64(20)$ & $211(17)$ & \\
\hline \multicolumn{4}{|l|}{ Tumor stage } \\
\hline 0 & $10(3)$ & $36(3)$ & .990 \\
\hline I & $99(32)$ & $412(34)$ & \\
\hline II & $124(40)$ & $484(40)$ & \\
\hline III & $55(18)$ & $196(16)$ & \\
\hline IV & $22(7)$ & $71(6)$ & \\
\hline \multicolumn{4}{|l|}{ Comorbidities } \\
\hline Induction chemotherapy & 137 (43) & $445(36)$ & .032 \\
\hline COPD & $41(13)$ & $98(8)$ & .011 \\
\hline Diabetes & $32(10)$ & $167(14)$ & .092 \\
\hline Congestive heart failure & $7(2)$ & $34(3)$ & .697 \\
\hline Myocardial infarction & $5(2)$ & $10(1)$ & .212 \\
\hline Peripheral vascular disease & $8(3)$ & $18(1)$ & .220 \\
\hline
\end{tabular}

software (version 3.1.2; R Foundation for Statistical Computing, Vienna, Austria). The University of Virginia Institutional Review Board for Health Sciences Research approved this study.

\section{RESULTS}

The SEER-Medicare database included 1688 patients who underwent esophagectomy among patients diagnosed with esophageal cancer from 2000 to 2009. Overall 30-day postoperative mortality was 116 of 1688 (6.9\%), and 90-day mortality was 234 of $1688(13.9 \%)$. After excluding in-hospital deaths-these patients are not candidates for readmission - the readmission analysis dataset included 1543 patients. Within this cohort, 90-day mortality after successful discharge was 98 of 1543 (6.4\%). Patient demographics are presented in Table 1.

The rate of readmission within 30 days of discharge was 319 of $1543(20.7 \%)$. The median readmission LOS was 4 days (interquartile range [IQR] 2-8 days), and most readmissions occurred within 15 days of discharge (Figure 1). Among readmitted patients, the in-hospital mortality rate was 28 of $319(8.8 \%)$. The 90 -day mortality rate among readmitted patients was almost 5 times that of nonreadmitted counterparts (52 of 319 [16.3\%] vs 46 of 1224 [3.8\%], $P<.001)$. Patients who were readmitted $\geq 2$ times did not have a significantly higher mortality rate

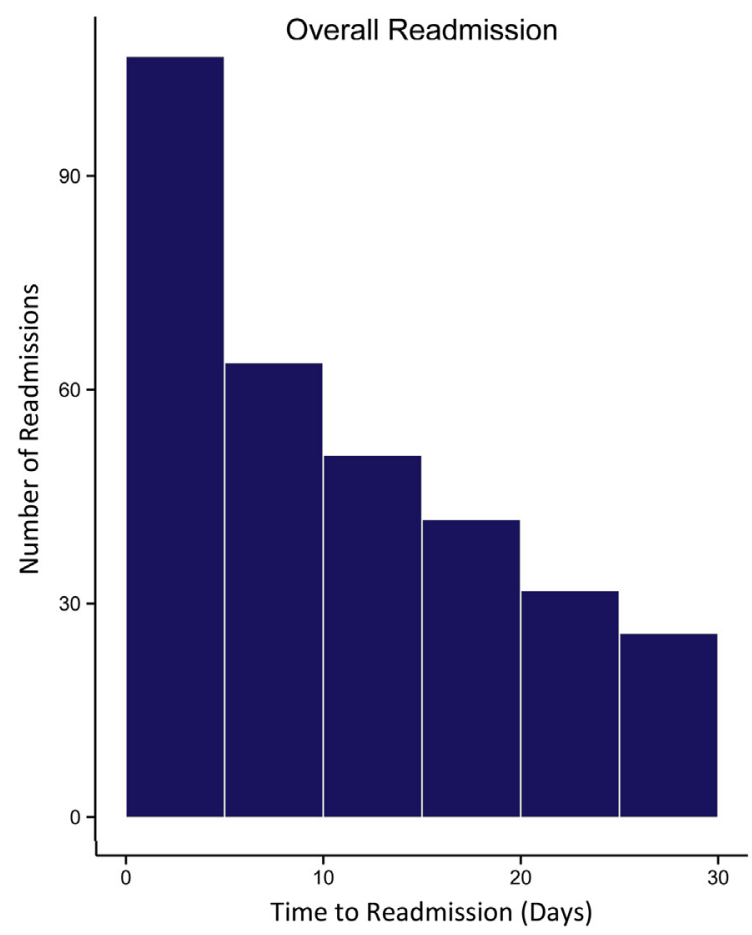

FIGURE 1. Distribution of readmissions over time: Postoperative readmissions were most common within the first 2 weeks of discharge.

(16 of $84[19.1 \%], 95 \%$ confidence interval [CI]: $12.0 \%-28.8 \%)$.

Multivariable analysis demonstrated postoperative readmission to be the strongest contributor to predicting mortality risk (odds ratio [OR] 6.64, $P<.001$; F test statistic: 33.5 ). The model C-statistic was 0.715 (Table 2). Provider covariance was not significant $(P=.072)$, suggesting that risk-adjusted mortality rates did not significantly differ across providers.

More than one third of readmissions were to facilities that did not perform the index operation (107 of 319 [33.5\%]). Readmissions to nonoperative hospitals were significantly more likely to undergo subsequent transfer $(15.0 \%$ vs $1.9 \%, P<.001)$. The 90 -day mortality did not differ

TABLE 2. Hierarchic generalized linear model for 90-day mortality

\begin{tabular}{lllrr}
\hline \multicolumn{1}{c}{ Variable } & OR & \multicolumn{1}{c}{ CI } & $\boldsymbol{P}$ value & F test \\
\hline Readmission & 6.64 & $3.49-12.62$ & $<.001$ & 33.47 \\
Age (10-y increment) & 2.20 & $1.29-3.75$ & $<.001$ & 8.50 \\
Discharge to home & 0.44 & $0.22-0.88$ & .019 & 5.48 \\
Induction chemotherapy & 1.59 & $0.84-2.99$ & .151 & 2.06 \\
Charlson-Deyo score & 0.83 & $0.52-1.34$ & .448 & 0.58 \\
Gender, female & 0.72 & $0.35-1.49$ & .372 & 0.80 \\
Surgery year & 0.97 & $0.82-1.16$ & .747 & 0.10 \\
Postoperative LOS (d) & & & & \\
$\quad<20$ & 1.04 & $0.97-1.12$ & .291 & 1.12 \\
$\quad 20$ & 0.96 & $0.88-1.05$ & .376 & 0.78 \\
\hline
\end{tabular}

$O R$, Odds ratio; $C I$, confidence interval; $L O S$, Length of stay. 
TABLE 3. Subgroup analysis: 90-day mortality

\begin{tabular}{lcc}
\hline \multicolumn{1}{c}{ Readmission diagnosis } & n & 90-day mortality (\%) \\
\hline Pulmonary & 114 & 22.8 \\
Pneumonia & & \\
Pleural effusion & & \\
Aspiration pneumonitis & \\
Other & 79 & \\
Gastrointestinal & & \\
Malnutrition/dehydration & \\
Dysphagia & & \\
Other gastrointestinal (including leak) & & \\
Other & 129 & \\
Cardiovascular & & \\
Infections (nonpulmonary) & \\
Wound \\
Other (including bleeding, AKI)
\end{tabular}

significantly between those patients readmitted to the operative facility and those readmitted to an alternate facility $(13.1 \%$ vs $17.9 \%, P=.269)$, nor did it differ between transferred and nontransferred patients $(20.0 \%$ vs $16.1 \%, P=.644)$. The most-frequent readmitting diagnoses were pneumonia $(11.8 \%)$, malnutrition/ dehydration $(8.1 \%)$, pleural effusion $(7.5 \%)$, and aspiration pneumonitis $(6.8 \%)$. Mortality at 90 days grouped by cause of readmission is presented in Table 3 . Notably, $>1$ in 5 patients readmitted with a pulmonary diagnosis died within 90 days of the operation. Temporal trends indicate that postoperative readmissions remained common up to 4 weeks after discharge (Figure 1).

The hierarchic logistic regression model used to estimate the risk of 30-day readmission obtained a C-statistic of 0.648. To account for the clustering effect of procedures within hospitals, the hospital provider was included as a random effect. The only significant preoperative predictor of readmission was delivery of induction therapy (OR $1.46, P=.032$ ) (Table 4 ). As was the case for mortality, the test for provider covariance was not significant $(P=.159)$, suggesting that risk-adjusted readmission rates did not differ significantly across providers.

TABLE 4. Hierarchic generalized linear model for 30-day readmission

\begin{tabular}{lcccc}
\hline \multicolumn{1}{c}{ Variable } & OR & CI & $\boldsymbol{P}$ value & F test \\
\hline Induction chemotherapy & 1.46 & $1.03-2.06$ & .032 & 4.64 \\
Discharge to home & 0.79 & $0.52-1.19$ & .257 & 1.29 \\
Gender, female & 1.24 & $0.84-1.83$ & .275 & 1.19 \\
Charlson-Deyo score & 1.14 & $0.89-1.46$ & .309 & 1.04 \\
Surgery year & 0.96 & $0.88-1.06$ & .421 & 0.65 \\
Age (10-y increment) & 0.99 & $0.73-1.36$ & .974 & 0 \\
Postoperative LOS (d) & & & & \\
$\quad<20$ & 1.02 & $0.98-1.06$ & .373 & 0.80 \\
$\quad \geq 20 \mathrm{~d}$ & 0.99 & $0.94-1.04$ & .671 & 0.18 \\
\hline
\end{tabular}

$O R$, Odds ratio; $C I$, confidence interval; $L O S$, length of stay.
We conducted a separate analysis of the association between LOS for the index operative hospitalization and readmission. The median LOS was 13 days (IQR: 9-20 days), and the most common LOS was 9 days. Logistic regression was used to assess the effect of LOS on readmission, with the effect of LOS modeled using natural cubic splines. The readmission probability for a 73-year-old patient (median age) with a Charlson index of 0 , who did not receive induction therapy, is shown in Figure 2; for an LOS of 9 days, we estimate a $12.6 \%$ chance of readmission. In general, the probability of readmission increases with increasing postoperative LOS (Figure 2). However, a deviation from this relationship occurred between a LOS of 9 and 23 days, with a peak at a LOS of 15 days. This finding suggests that patients have an uncharacteristically elevated risk of readmission over a range of index hospitalization lengths.

\section{DISCUSSION}

In this study, we used a large, national dataset to examine the relationship between postoperative readmissions and subsequent 90-day mortality among patients undergoing esophagectomy for cancer. Predictably, early readmissions are very common after esophagectomy. A total of $33.5 \%$ of readmissions were to facilities that did not perform the index operation, an indication that quality metrics that focus on readmissions to the index hospital may substantially

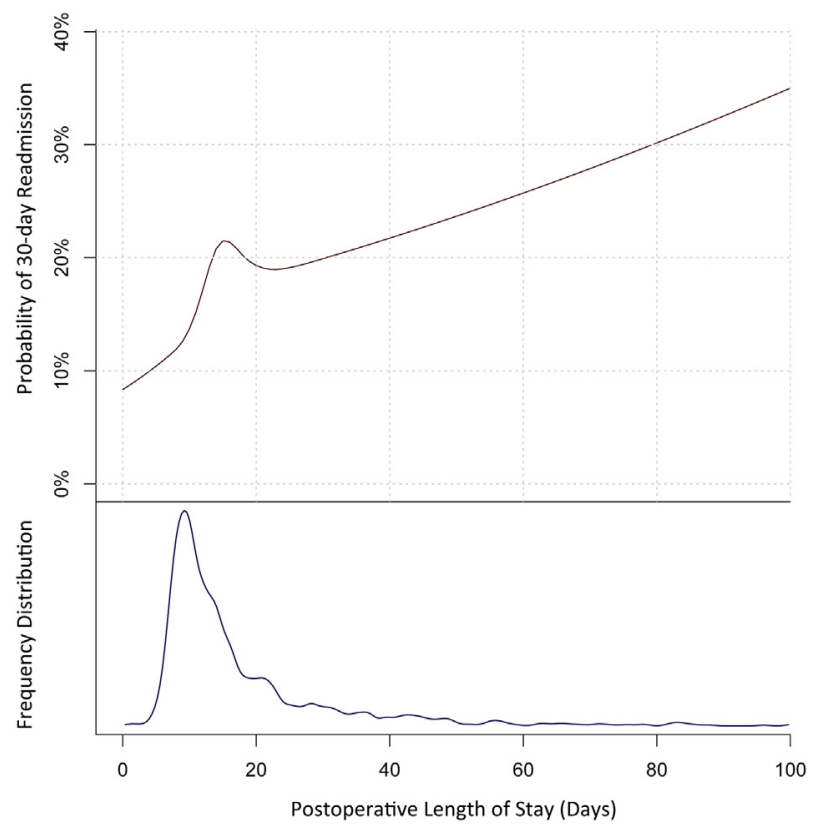

FIGURE 2. Readmission rate versus postoperative LOS: The probability of readmission within 30 days is higher for patients with prolonged postoperative LOS (top). For the mode LOS (9 days), the readmission rate is relatively low, at $12.6 \%$. The frequency distribution of postoperative LOS is provided for comparison (bottom). 
underestimate readmission rate. Moreover, not all readmissions have the same clinical significance. Among major organ systems, pulmonary complications are responsible for the largest proportion of readmissions, and portend the worst clinical outcome. Our results highlight the acuity of this extremely high-risk population, and argue for early and intensive medical attention.

We found that early readmission outperforms all commonly reported preoperative risk factors in predicting mortality after esophagectomy. Because readmission is a postoperative risk factor, this associative finding was not entirely unexpected. However, the magnitude of the risk increase was notable, as readmitted patients had a nearly 5 -fold increase in mortality rate within the subsequent 2 to 3 months. Risk for early mortality after readmission was high, regardless of the readmitting hospital or patient transfer status. In a recent study, Fernandez and colleagues $^{22}$ reported that early readmission after esophagectomy portends a negative long-term prognosis. Our results indicate that much of this impact is felt within the first 3 months after discharge.

Analyses by readmitting diagnosis suggest that readmissions related to pulmonary complications carry the worst short-term prognosis. This finding is corroborated by prior work demonstrating negative associations between pulmonary complications, and both short- and long-term survival after esophagectomy. ${ }^{23,24}$ Although these studies focused on in-hospital complications, ours is the first to demonstrate that pulmonary readmissions within 30 days of discharge are equally ominous. Patients with nonspecific dyspneic symptoms or small pleural effusions should receive aggressive care upon readmission, as $>20 \%$ will not survive the next few months.

These results reinforce the notion that a fairly benign readmitting diagnosis is often an indicator of a much more severe root process. For example, malnutrition and dehydration carried the highest mortality rate of any nonpulmonary readmission diagnosis. Among patients undergoing esophagectomy, nutritional status is often compromised to some degree preoperatively through induction treatment. ${ }^{25}$ This factor may explain the observation in our dataset that induction therapy was a predictor of both readmission and early mortality. Although many interventions can promote postoperative nutrition, ${ }^{26-28}$ a readmission owing to poor dietary tolerance often indicates other complications, such as infection, stenosis, or anastomotic leak. Clearly, the theme that an initial readmitting diagnosis may not indicate the root cause of decompensation is pervasive. A thorough root-cause analysis should be part of every readmission process.

The most common postoperative LOS for patients in our study was 9 days. Overall, those who stayed longer tended to have a higher risk of readmission. This trend indicates that prolonged LOS may be related to unforeseen complications or sicker patients. An encouraging finding was that a 9-day LOS in an otherwise healthy patient was associated with a comparatively low readmission rate (12.6\%; Figure 2). Thus, patients discharged after a routine hospital course are relatively unlikely to be readmitted.

We found that the relationship between index LOS and readmission deviated from an otherwise relatively linear pattern between the range of 9 to 23 days, with a peak at index LOS 15 days. Although the precise range of LOS that was associated with elevated readmission risk varied slightly depending on spline knot placement, we found that other knot placements produced very similar results. Freeman and colleagues ${ }^{29}$ previously reported similar findings with pulmonary lobectomy. These results suggest that, whereas the routine esophagectomy patient may be discharged expeditiously, those who have already experienced a relatively prolonged postoperative course (15 days) may benefit from additional observation. Early results from enhanced recovery programs after surgery report shorter LOS without deleterious effects on readmission rate. ${ }^{6,30}$ Results from our study advocate for careful management of patients who deviate from the standard postoperative recovery pathway.

Despite its clinical relevancy, the validity of readmission as a quality indicator is difficult to establish. In a previous study, we found that risk-adjusted readmission rate after lung cancer resection does not vary significantly across hospital providers. ${ }^{11}$ This study further shows that riskadjusted readmission rate after esophagectomy additionally does not significantly vary across providers. The incentive structure associated with adopting readmission as a quality metric is problematic. Varghese and colleagues ${ }^{12}$ previously demonstrated that hospitals qualifying for "leapfrog" case-volume criteria for esophagectomy had lower odds of 90-day mortality, compared with nonqualifying hospitals, yet had higher odds of readmission. This finding may be explained by a potential tendency among leapfrog-qualifying hospitals to provide more clinically appropriate readmissions after complicated procedures.

An earlier study using American College of Surgeons National Surgical Quality Improvement Program showed similar complication rates between high-and low-mortality providers, but marked differences in mortality rates associated with each complication. ${ }^{9}$ These findings suggest that appropriate management of complications - more so than prevention-is the strongest determinant of mortality outcomes. Home interventions, nutritional counseling, early provider-initiated telephone follow-up, and other discharge planning strategies have been shown to reduce readmissions and emergency department visits among medically managed patients. ${ }^{31,32}$ Results in the surgical population are thus far unclear; a recent multicenter trial instituting centralized telephonebased counseling after colorectal resection failed to 
demonstrate an impact on readmission and quality of life. ${ }^{33}$ Conversely, Nabagiez and colleagues ${ }^{34}$ recently reported that a physician-assistant home care program was associated with a reduction in the incidence of infectionrelated readmissions after cardiac surgery.

This study has several limitations. The SEER-Medicare dataset, by excluding patients aged $<65$ years, is older than the general esophagectomy population. However, the peak incidence of esophageal cancer occurs in patients aged 75 to 85 years $^{35}$; therefore, our results are relevant to most patients who are undergoing esophagectomy for cancer. Second, SEER-Medicare does not clearly separate preoperative from postoperative diagnosis codes. Therefore, we could not incorporate postoperative complications into our predictive models of readmission or 90-day mortality. However, SEER-Medicare reliably captures readmissions to any facility and long-term mortality, making it a relevant source of data for the present study's purpose. When performing risk analysis with administrative databases, selecting only reliably captured variables for modeling is critical.

Third, the study may not adequately represent patients who have an extreme index postoperative LOS, because 90-day mortality was calculated from the date of surgery, rather than the date of discharge. Finally, causes of readmission were based on ICD-9 diagnosis codes. These codes are often recorded early during an admission. Despite application of investigator judgement, these codes may not always represent the root complication responsible for each readmission. For example, only 6 readmission diagnoses were indicative of anastomotic leak, which is a complication frequently not discovered until $\geq 1$ days into a readmission.

\section{CONCLUSIONS}

Early readmissions are very common after esophagectomy for cancer, especially among patients who underwent induction chemotherapy. Patients who deviate from a standard postoperative course and are considered for discharge after an index hospitalization of 15 days are at particular risk for early readmission. Readmitted patients need to be recognized for their high risk of early mortality. In particular, 1 in 5 patients readmitted with a pulmonary diagnosis will die within 90 days of surgery. Regardless of their symptoms, all patients readmitted after esophagectomy should undergo an exhaustive root-cause exploration, intensive monitoring, and aggressive care.

\section{Conflict of Interest Statement}

Authors have nothing to disclose with regard to commercial support.

\section{References}

1. Edwards BK, Ward E, Kohler BA, Eheman C, Zauber AG, Anderson RN, et al Annual report to the nation on the status of cancer, 1975-2006, featuring colorectal cancer trends and impact of interventions (risk factors, screening, and treatment) to reduce future rates. Cancer. 2010;116:544-73.

2. Swisher SG, Deford L, Merriman KW, Walsh GL, Smythe R, Vaporicyan A, et al. Effect of operative volume on morbidity, mortality, and hospital use after esophagectomy for cancer. J Thorac Cardiovasc Surg. 2000;119:1126-32.

3. Lapar DJ, Stukenborg GJ, Lau CL, Jones DR, Kozower BD. Differences in reported esophageal cancer resection outcomes between national clinical and administrative databases. J Thorac Cardiovasc Surg. 2012;144:1152-7.

4. Luketich JD, Pennathur A, Awais O, Levy RM, Keeley S, Shende M, et al Outcomes after minimally invasive esophagectomy: review of over 1000 patients. Ann Surg. 2012;256:95-103.

5. Boshier PR, Anderson O, Hanna GB. Transthoracic versus transhiatal esophagectomy for the treatment of esophagogastric cancer: a meta-analysis Ann Surg. 2011;254:894-906.

6. Markar SR, Karthikesalingam A, Low DE. Enhanced recovery pathways lead to an improvement in postoperative outcomes following esophagectomy: systematic review and pooled analysis. Dis Esophagus. 2015;28:467-75.

7. Walters DM, McMurry TL, Isbell JM, Stukenborg GJ, Kozower BD. Understanding mortality as a quality indicator after esophagectomy. Ann Thorac Surg. 2014;98:506-11; discussion 511-2.

8. Luc G, Durand M, Chiche L, Collet D. Major post-operative complications predict long-term survival after esophagectomy in patients with adenocarcinoma of the esophagus. World J Surg. 2015;39:216-22.

9. Ghaferi AA, Birkmeyer JD, Dimick JB. Variation in hospital mortality associated with inpatient surgery. N Engl J Med. 2009;361:1368-75.

10. Lum HD, Studenski SA, Degenholtz HB, Hardy SE. Early hospital readmission is a predictor of one-year mortality in community-dwelling older Medicare beneficiaries. J Gen Intern Med. 2012;27:1467-74.

11. Hu Y, McMurry TL, Isbell JM, Stukenborg GJ, Kozower BD. Readmission after lung cancer resection is associated with a 6-fold increase in 90-day postoperative mortality. J Thorac Cardiovasc Surg. 2014;148:2261-7.

12. Varghese TK Jr, Wood DE, Farjah F, Oelschlager BK, Symons RG, MacLeod KE, et al. Variation in esophagectomy outcomes in hospitals meeting leapfrog volume outcome standards. Ann Thorac Surg. 2011;91:1003-9; discussion 1009-10.

13. Goodney PP, Stukel TA, Lucas FL, Finlayson EV, Birkmeyer JD. Hospital volume, length of stay, and readmission rates in high-risk surgery. Ann Surg. 2003;238:161-7.

14. Wang H, Shen Y, Feng M, Zhang Y, Jiang W, Xu S, et al. Outcomes, quality of life, and survival after esophagectomy for squamous cell carcinoma: a propensity score-matched comparison of operative approaches. J Thorac Cardiovasc Surg. 2015;149:1006-14; discussion 1014-5.e4.

15. Schneider EB, Hyder O, Brooke BS, Efron J, Cameron JL, Edil BH, et al. Patient readmission and mortality after colorectal surgery for colon cancer: impact of length of stay relative to other clinical factors. J Am Coll Surg. 2012;214: 390-8; discussion 398-9.

16. Schneider EB, Hyder O, Wolfgang CL, Hirose K, Choti MA, Makary MA, et al Patient readmission and mortality after surgery for hepato-pancreato-biliary malignancies. J Am Coll Surg. 2012;215:607-15.

17. Warren JL, Klabunde CN, Schrag D, Bach PB, Riley GF. Overview of the SEER-medicare data: content, research applications, and generalizability to the United States elderly population. Med Care. 2002;40(8 Suppl):IV-3-18.

18. Deyo RA, Cherkin DC, Ciol MA. Adapting a clinical comorbidity index for use with ICD-9-CM administrative databases. J Clin Epidemiol. 1992;45:613-9.

19. Klabunde CN, Potosky AL, Legler JM, Warren JL. Development of a comorbidity index using physician claims data. J Clin Epidemiol. 2000;53:1258-67.

20. National Cancer Institute. SEER-Medicare: calculation of comorbidity weights 2010. Available at: http://healthservices.cancer.gov/seermedicare/program/ comorbidity.html. Accessed October 15, 2014.

21. Hu Y, McMurry TL, Wells KM, Isbell JM, Stukenborg GJ, Kozower BD. Postoperative mortality is an inadequate quality indicator for lung cancer resection. Ann Thorac Surg. 2014;97:973-9.

22. Fernandez FG, Khullar O, Force SD, Jiang R, Pickens A, Howard D, et al Hospital readmission is associated with poor survival after esophagectomy for esophageal cancer. Ann Thorac Surg. 2015;99:292-7.

23. Ferguson MK, Durkin AE. Preoperative prediction of the risk of pulmonary complications after esophagectomy for cancer. J Thorac Cardiovasc Surg. 2002;123:661-9.

24. Schieman C, Wigle DA, Deschamps C, Nichols Iii FC, Cassivi SD, Shen KR, et al. Patterns of operative mortality following esophagectomy. Dis Esophagus. 2012;25:645-51. 
25. Sikora SS, Ribeiro U, Kane JM III, Landreneau RJ, Lembersky B, Posner MC Role of nutrition support during induction chemoradiation therapy in esophageal cancer. JPEN J Parenter Enteral Nutr. 1998;22:18-21.

26. Baker A, Wooten LA, Malloy M. Nutritional considerations after gastrectomy and esophagectomy for malignancy. Curr Treat Options Oncol. 2011;12: $85-95$.

27. Xiao-Bo Y, Qiang L, Xiong Q, Zheng R, Jian Z, Jian-Hua Z, et al. Efficacy of early postoperative enteral nutrition in supporting patients after esophagectomy. Minerva Chir. 2014;69:37-46.

28. Yamasaki M, Miyata H, Yasuda T, Shiraishi O, Takahashi T, Motoori M, et al Impact of the route of reconstruction on post-operative morbidity and malnutrition after esophagectomy: a multicenter cohort study. World J Surg. 2015;39: 433-40.

29. Freeman RK, Dilts JR, Ascioti AJ, Dake M, Mahidhara RS. A comparison of length of stay, readmission rate, and facility reimbursement after lobectomy of the lung. Ann Thorac Surg. 2013;96:1740-5; discussion 1745-6.

30. Shewale JB, Correa AM, Baker CM, Villafane-Ferriol N, Hofstetter WL, Jordan VS, et al. Impact of a fast-track esophagectomy protocol on esophageal cancer patient outcomes and hospital charges. Ann Surg. 2015; 261:1114-23.
31. Costantino ME, Frey B, Hall B, Painter P. The influence of a postdischarge intervention on reducing hospital readmissions in a medicare population. Popul Health Manag. 2013;16:310-6.

32. Dudas V, Bookwalter T, Kerr KM, Pantilat SZ. The impact of follow-up telephone calls to patients after hospitalization. Am J Med. 2001;111: 26S-30S.

33. Young JM, Butow PN, Walsh J, Durcinoska I, Dobbins TA, Rodwell L, et al. Multicenter randomized trial of centralized nurse-led telephone-based care coordination to improve outcomes after surgical resection for colorectal cancer: the CONNECT intervention. J Clin Oncol. 2013;31:3585-91.

34. Nabagiez JP, Shariff MA, Khan MA, Molloy WJ, McGinn JT Jr. Physician assistant home visit program to reduce hospital readmissions. J Thorac Cardiovasc Surg. 2013;145. 225-231, 233; discussion 232-3.

35. Howlader N, Noone A, Krapcho M, Neyman N, Aminou R, Waldron W, et al SEER cancer statistics review, 1975-2008. Bethesda, MD: National Cancer Institute; 2011

Key Words: esophageal cancer, esophagectomy, postoperative readmission, postoperative mortality, outcomes

Readers who found these articles interesting may also like to read the following papers found in recent and future issues of our sister publications, Seminars in Thoracic and Cardiovascular Surgery and Operative Techniques in Thoracic and Cardiovascular Surgery!

\section{General Thoracic Surgery: Other Thoracic Surgical Papers}

Current Readings: Redefining Minimally Invasive Uniportal Video-Assisted Thoracic Surgery. David Zeltsman. Semin Thorac Cardiovasc Surg 2014; Autumn; 26(3):249-254.

Current Readings: Failed Hiatus Hernia Repair. Sumeet Mittal. Semin Thorac Cardiovasc Surg 2014; Winter; 26(4):331-334.

State of the Art: The ITMIG Thymic Initiative: A State of the Art for the Study of Thymic Malignancies. Frank Detterbeck. Semin Thorac Cardiovasc Surg 2014; Winter; 26(4):317-322.

State of the Art: The Theory, Practice and Future of Process Improvement in General Thoracic Surgery. Richard Freeman. Semin Thorac Cardiovasc Surg 2014; Winter; 26(4):310-316.

State of the Art: Using Clinical Risk Models for Lung Nodule Classification. Eric Grogan. Semin Thorac Cardiovasc Surg 2015; Spring; 27(1):30-35.

Original Submission: Eliminating a need for esophagectomy: endoscopic treatment of Barrett's Esophagus with early esophageal neoplasia. Thomas Watson. Semin Thorac Cardiovasc Surg 2014; Winter; 26(4):274-284.

Editorial Commentary: Endoscopically closing doors. Anthony Kim. Semin Thorac Cardiovasc Surg 2014; Winter; 26(4):285-286.

Original Submission: Incidence and risk factors for respiratory complications in patients undergoing esophagectomy for malignancy: a NSQIP analysis. Daniela Molena. Semin Thorac Cardiovasc Surg 2014; Winter; 26(4):287-294.

Editorial Commentary: Esophageal complications: What are the real results? R. Taylor Ripley. Semin Thorac Cardiovasc Surg 2014; Winter; 26(4):295-296

News and Views: Chest Wall Reconstruction. Calvin Ng. Expected Publication October 2015.

Current Readings: What the Surgeon Needs to Know About Databases. Alessandro Brunelli. Expected Publication October 2015.

Original Submission: Endobronchial Treatment of bronchopleural fistulas by using Intrabronchial valve system: a case series. Eitan Podgaetz. Expected Publication October 2015.

The Minimally Invasive Repair of Pectus Excavatum. Donald Nuss. Oper Tech Thorac Cardiovasc Surg 2014; Autumn; 19 (3):324-347. Total Pectus Excavatum Repair: Open Approach. John Kucharczuk. Oper Tech Thorac Cardiovasc Surg 2014; Autumn; 19(3):348-364. Minimally Invasive Resection of Benign Esophageal Lesions - Katie S. Nason. Oper Tech Thorac Cardiovasc Surg 2014; Winter, 19(4):396-413.

Video-assisted Thoracoscopic Bilobectomy. Puja Gaur. Oper Tech Throac Cardiovasc surg 2014; Winter, 19(4):414-432. 